\title{
CHAROPHYTA DEL CRETÁCICO TARDÍO Y EL PALEOCENO DEL CENTRO OESTE DE ARGENTINA
}

\author{
EDUARDO A. MUSACCHIO \\ Laboratorio de Bioestratigrafía, UNPSJB (9000), Comodoro Rivadavia, Argentina.aldo@unpbib.edu.ar
}

\begin{abstract}
RESUMO - Os carófitos do Cretáceo Superior da América do Sul meridional mostram um aumento relativo em sua diversidade e distribuição geográfica após o início do Campaniano, quando comparados com as associações prévias das mesmas bacias. As causas deste processo podem estar nas novas condições estruturais e geo-ambientais estabelecidas no continente no início do Campaniano. A partir do Paleoceno as carófitas da região centro-oeste da Argentina reduzem novamente sua diversidade e retornam às condições que exibiam antes do Campaniano, especialmente em nível de gênero.
\end{abstract}

Palabras-chave: Algas fósseis, Charophyta, Cretáceo, Paleoceno, América do Sul meridional.

\begin{abstract}
LATE CRETACEOUS AND PALEOCENE CHAROPHYTA FROM WEST CENTRAL ARGENTINA. Comparing with pre-Campanian assemblages, the charophytes of uppermost Cretaceous age from southern South America show a relative increase in their taxonomical diversity, as well as include taxa with wide geographical distribution. These both attributes, high diversity and wide distribution, can be explained in the frame of the new tectonic and geo-environmental conditions settled in the Continent at the early-Campanian. The Paleocene charophytes of west central Argentina include scarce novelties at the generic level and they seem return to rates of diversity that characterize others pre-Campanian floras for the same region.
\end{abstract}

Key words: Fossil algae, Charophyta, Cretaceous, Paleocene, Southern South America.

\section{INTRODUCCIÓN}

Los carófitos son un grupo de algas verdes conocidas en el registro fósil desde el Silúrico Tardío. Estas algas están, presentes en diferentes ambientes sedimentarios subacuáticos de poca profundidad, principalmente continentales. Los restos más estudiados dentro del registro fósil son las estructuras calcificadas en vida del talo y, entre éstas, los girogonites: diminutas piezas calcáreas que forman parte del gametangio femenino (Figura 1). En su primera parte, la presente contribución está dedicada al análisis de la diversidad taxonómica del registro fósil, principalmente en el centro-oeste de Argentina, durante el Cretácico Tardío. Estudios sistemáticos previos para esta región (Musacchio, 2000) permiten advertir la existencia de un máximo en el número de géneros y especies al término del período anotado. La amplia distribución geográfica de algunos de estos taxa durante el Campaniano y el Maastrichtiano sugiere, además, la reapertura de condiciones de libre intercambio florístico para América del Sur, reconocidas previamente durante el Aptiano (Musacchio, 2000). Las floras de carófitos neocretácicos del Grupo Baurú en Brasil (Petri, 1955; DiasBrito et al., 2001) permiten testar las evidencias recién mencionadas para los carófitos procedentes del centro-oeste de Argentina. Se analiza a continuación el registro disponible en el centro-oeste de Argentina para los caróftos paleocénicos, el que parece retornar a frecuencias similares, en cuanto la diversidad taxonómica, a las computadas para tiempos pre-campanianos. Por último, se discute la posible responsabilidad de factores geológicos externos en la regulación de los cambios paleobiogeográficos. Los cambios tectónicos ocurridos hacia el inicio del Campaniano permitirán comprender mejor las diferencias previamente enfatizadas sobre la diversidad.

\section{DATOS BIOESTRATIGRÁFICOS}

\section{La diversidad taxonómica hacia el Cretácico terminal}

En la Cuenca del Neuquén (centro oeste de Argentina) los carófitos neocretácicos conocidos hasta el presente proceden de entidades estratigráficas integrantes de los "Estratos con Dinosaurios" y de la Formación Loncoche (Musacchio, 1973; Uliana \& Musacchio, 1978; Musacchio, 2000). La antigüedad de las asociaciones procedentes de los "Estratos con Dinosaurios" tiene una referencia confiable en cuanto a sus límites extremos posibles: el inferior es de la antigüedad del Cenomaniano, el superior del Maastrichtiano. Las entidades intermedias cuentan con información de valor cronológico dispar para los diferentes casos.

En los "Estratos con Dinosaurios" [= Grupo Pehuenche Doering (nombre correcto), no Grupo Neuquén (nombre inválido; preocupado por Weaver, 1931)] se conocen hasta el presente tres asociaciones con carófitos encontrados, respectivamente, en las Formaciones Portezuelo (a), "Plottier" 


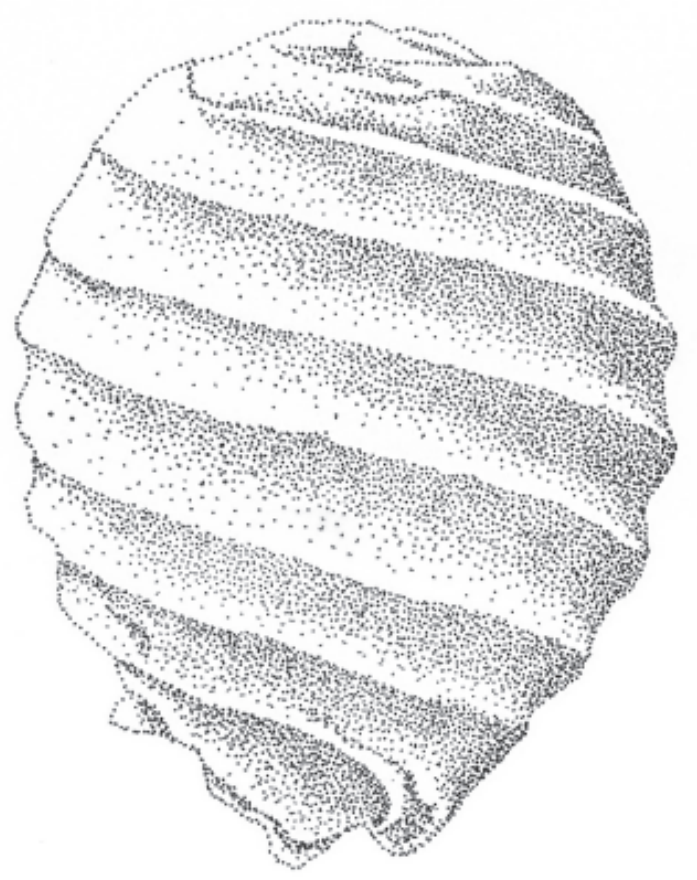

Figura 1. Girogonite de la especie viviente Lychnothamnus barbatus (Meyen), modificado de Soulie-Märsche (1989, pl. XXXV, fig. 1). LPA aproximadamente $950 \mu \mathrm{m}$.

Figure 1. Girogonite of modern Lychnothamnus barbatus (Meyen), modified from Soulie-Märsche (1989, pl. XXXV, fig. 1). LPA nearly $950 \mu \mathrm{m}$.

(b) y Anacleto (c), de abajo hacia arriba. El límite inferior máximo está dado por la asociación de palinomorfos correspondiente a la Zona de Fraxinoipollenites fragilis, asignada por Vallati (2001), al Albiano?-Cenomaniano. Se trata, esta última, de una asociación con angiospermas caracterizada por granos tricolpados, tricolporoidados y tricolporados, en la que faltan los triporados, procedente de la parte alta de la Formación Huincul. Esta entidad estratigráfica es integrante de los mencionados "Estratos con Dinosaurios" en El Zampal, e infrayace a la Formación Portezuelo dentro de la misma cuenca. Por su parte, la Formación Lonchoche con carófitos (d) es del Maastrichtiano, alcanzando en partes el Paleoceno como se detallará abajo.

Los carófitos conocidos para la Formación Portezuelo son Peckisphaera portezueloensis Musacchio, 1973 y Mesochara ameghinoi Musacchio, 1973. Estos girogonites y los ostrácodos asociados no han aportado, sobre la base del material disponible, criterios de correlación firme y han sido asignados, en forma tentativa, al intervalo TuronianoSantoniano por Musacchio (2000).

Las fangolitas (mudstones) de color rojo-ladrillo en El Zampal, Provincia de Mendoza (Figura 2), muy parecidas a las que son conspicuas para la Formación Plottier en otras partes de la cuenca (Cazau \& Uliana, 1973), contienen una asociación de Lychnothamnus barbosai nov. comb. Ilyocypris riograndensis. Esta asociación es casi siempre monotípica en cuanto a los carófitos, aunque incluye numerosas especies de ostrácodos no marinos. La misma

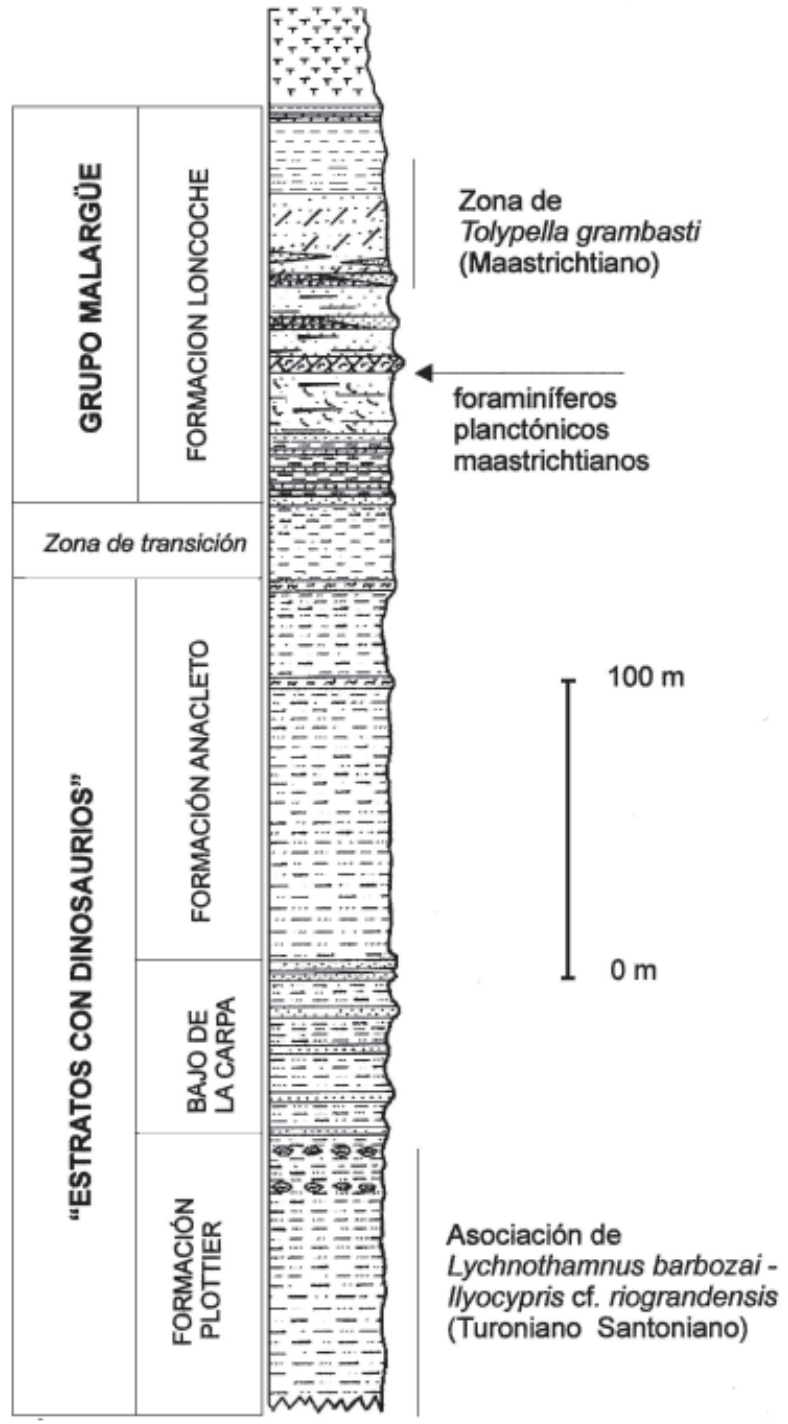

Figura 2. Sección columnar en El Zampal, parte superior, Provincia de Mendoza, Centro-oeste de Argentina.

Figure 2. Geological section of the upper part of El Zampal succession, Mendoza, West-central Argentina.

se compara, sin dificultades, con aquella similar conocida en Brasil para la Formación Adamantina en São Paulo y Minas Gerais bajo el nombre de Asociación de "Chara" barbosai - Ilyocypris cf. riograndensis (Figura 3), la que fuera asignada al intervalo Turoniano-Santoniano (Musacchio in Dias-Brito et al., 1998; Musacchio in DiasBrito et al., 2001).

En El Zampal, el ostrácodo Ilyocypris riograndensis Musacchio \& Simeoni, 1990, sensu lato, caracteriza la subzona homónima y está presente en una sección de sedimentos lacustres, la que fuera correlacionada con el conjunto de las Formaciones Plottier, Bajo de la Carpa y Anacleto. Los mismos autores, Musacchio \& Simeoni (1990:358), asignaron este intervalo donde está registrada I. riograndensis nov. sp. al Senoniano, pre-Maastrichtiano. El material recuperado de Lychnothamnus barbosai (Petri, 1955) nov. comb. es de 

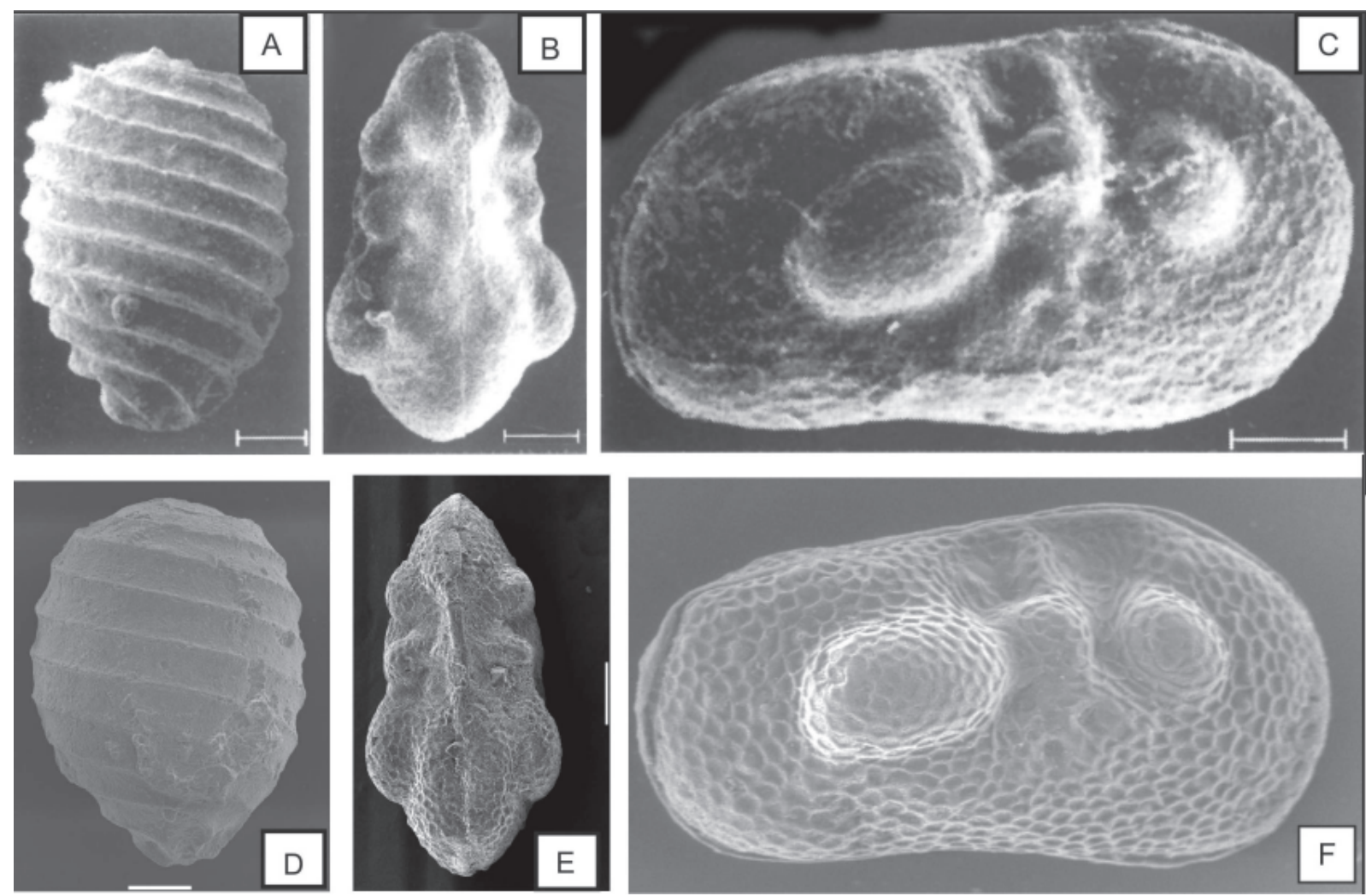

Figura 3. Marcadores de la Asociación Lychnothamnus barbosai - Ilyocypris cf. riograndensis (Turoniano-Santoniano) de la Formación Adamantina, Brasil (A-C) y de la Formación Plottier, Argentina (D-F): A, D, Lychnothamnus barbosai (Charophyta), con girogonites en vista lateral; B, C, E, F, llyocypris cf. riograndensis (Ostracoda). B, E: caparazones en vista dorsal; C, F: caparazones en vista lateral derecha. Escala $=0,1 \mathrm{~mm}$.

Figure 3. Main elements from the Lychnothamnus barbosai - Ilyocypris cf. riograndensis (Turoniano-Santoniano) Association, from Adamantina Formation in Brasil (A-C) and Plottier Formation in Argentina (D-F): A, D, Lychnothamnus barbosai (Charophyta), lateral view of the girogonites; B, C, E, F, llyocypris cf. riograndensis (Ostracoda). B, E: dorsal view; C, F: right lateral view. Scale bar $=0.1 \mathrm{~mm}$

aproximadamente 120 girogonites para cuatro muestras de la sección "Plottier" (de color predominante: 5 P 5/4, USA-GS Color Chart). Este número es relativamente escaso si se lo compara con el registro brasileño, el que cuenta con varios miles de girogonites para el Estado de São Paulo. Estos últimos proceden, mayormente, de limolitas rojizas y castañorojizas. Los girogonites de L. barbosai en El Zampal muestran células espirales generalmente más calcificados que los de Brasil. Junto a los mismos, en algunas muestras de El Zampal, aparece un morfotipo con girogonites más grandes y más globosos, claramente co-genéricos con los primeros, el cual no ha sido observado en las floras de la Formación Adamantina. La presencia del género Lychnothamnus (Ruprecht) Leonhardi emend. A. Braun en asociaciones sudamericanas del Turoniano-Santoniano, caracterizadas por el aislamiento geográfico de sus integrantes, plantea un interesante problema biogeográfico, sobre todo por la semejanza de la especie sudamericana con Lychnothamnus barbatus, especie viviente protegida en algunos países por su carácter relictual (Soulie-Märsche, 1989; Soulie-Märsche \& Martin-Closas, 2003; García, 2003). El abordaje del problema antes anotado escapa, sin embargo, al tema de la presente contribución.

Hacia arriba, la Formación Anacleto está formada por pelitas varicolores que representan un episodio lacustre muy conspicuo, ampliamente distribuido en la cuenca, en el que los carófitos y los ostrácodos continentales son muy abundantes. Esta entidad ha sido asignada, al menos en parte, al Campaniano (Musacchio \& Simeoni, 1991). Los carófitos descriptos (Musacchio, 1973) han sido incluidos en Platychara, Notochara, Chara?, Gobichara (Pseudoharrisichara), a los que debería agregarse Lychnothamnus y Mesochara.

Los carófitos cretácicos más modernos pertenecen a un intervalo con intercalaciones de areniscas, limolitas y tobas arenosas marinas de la Formación Loncoche en Zampal (Provincia de Mendoza). Estas intercalaciones marinas son portadoras de foraminíferos, los que permiten corroborar la asignación cronológica de la flora contenida en los niveles no marinos intercalados al Maastrichtriano (Musacchio y Simeoni, 1990). La flora de carófitos incluye taxa de algunos géneros con amplia distribución geográfica tales como: Platychara, Tolypella, Feistiella, Gobichara (Pseudoharrisichara), Lamprothamnium, Chara.

A continuación parece de interés hacer una breve mención de algunos taxa asignados a estos géneros, destacando sus afinidades con formas descriptas previamente en otros continentes (Figura 4). 


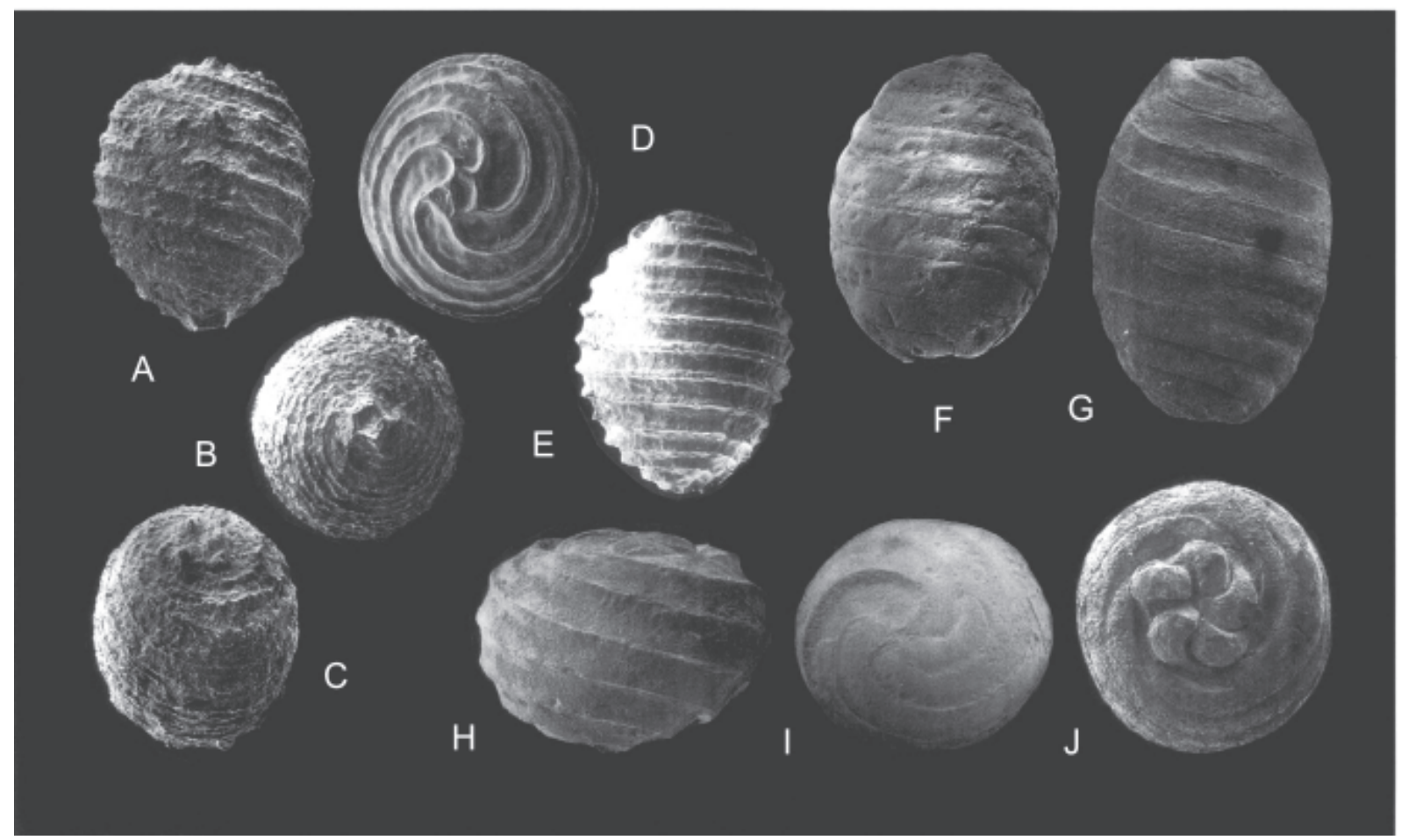

Figura 4. Girogonites maastrichtianos representativos de la Formación Loncoche en Mendoza, Argentina: A-C, Gobichara (Pseudoharrisichara) groeberi en vista lateral (A), basal (B) y apical inclinada (C), mismo espécimen, LPA = $810 \mu \mathrm{m}$.; D-E, Tolypella grambasti en vista basal $(D, L E D=240 \mu \mathrm{m})$ y lateral $(E, L P A=305 \mu \mathrm{m}) ; F-G$, Feistiella anluensis (diferentes girogonites en vista lateral $(F$, $\mathrm{LPA}=880 \mu \mathrm{m} ; \mathrm{G}, \mathrm{LPA}=1005 \mu \mathrm{m}) ; \mathrm{H}-\mathrm{J}$, Platychara perlata en vista lateral $(\mathrm{H}, \mathrm{LPA}=940 \mu \mathrm{m})$, basal $(\mathrm{I}, \mathrm{LED}=1060 \mu \mathrm{m})$ y apical $(\mathrm{J}, \mathrm{LED}=$ $925 \mu \mathrm{m})$; tres girogonites de diferentes procedencias.

Figure 4. Maastrichtian girogonites from the Lancoche Formation, Mendoza, Argentina: A-C, Gobichara (Pseudoharrisichara) groeberi in lateral (A), basal (B) and inclined apical (C) view, same specimen; D-E, Tolypella grambasti in basal (D) and lateral (E) views; F-G: Feistiella anluensis, distinct girogonites in lateral view; H-J, Platychara perlata in lateral $(\mathrm{H})$, basal $(\mathrm{I})$ and apical views; three girogonites from distinct places.

Platychara perlata (Peck \& Reker, 1947) es conocida en Argentina para las Provincias de Jujuy (Figura 5) y Mendoza (Musacchio \& Moroni, 1983:22-23). Formas similares, aunque con diferentes estados de oblamiento (LPA/LED), han sido registradas en Asia y América del Norte para el Cretácico terminal y para el Paleógeno. Parece existir una tendencia en el tiempo al oblamiento creciente, como así también al aumento de la talla (Tablas 1 y 2). Ésta última está basada en mediciones de girogonites maastrichtianos [(Uliana \& Musacchio, 1978:129 en Platychara cruciana (Horn av Rantzien), nombre ahora trasladado)] y paleocénicos (Musacchio \& Moroni, 1983:22).

Tolypella grambasti Musacchio (in Uliana \& Musacchio, 1978: 131-132), especie marcadora de la zona homónima de Musacchio (1989), ha sido identificado en Canadá por Feist (comunicación personal). Feistiella anluensis nov. comb. (para Porochara anluensis Wang, ver Wang, 1978) es una porocharacea de gran talla, representada en El Zampal por un registro de 390 girogonites. Estos han sido descriptos por Musacchio (in Uliana \& Musacchio, 1978:128), bajo Porochara cf gildemeisteri Koch \& Blisenbach. Sin embargo, esta semejanza es distante cuando se comparan los girogonites de El Zampal con Feistiella anluensis, una especie propuesta originalmente en China (ver observaciones, distribución, y bibliografía en Li, 1998: 99-100). Por su parte,
Kumar \& Grambast-Fessard (1984:254: est. I; 1-2, 4-5, 7) han descripto e ilustrado para Jamaica, una especie comparable bajo Porochara anluensis Wang.

La especie Gobichara (Pseudoharridsichara) groeberi Musacchio (in Uliana \& Musacchio, 1978:129) tiene semejanzas al nivel genérico con especies propuestas por Karczeewska \& Ziembinska-Tworzylo (1972) dentro de Gobichara nov. gen. Y, en particular, con G. nigra Karczeewska \& Ziembinska-Tworzylo. (1972:15), descripta para el Cretácico Superior de Mongolia. Asimismo, se parece a Microchara cristata Grambast (1971:35-37, pl.28, 29 y fig. 22), del Maastrichtiano de Rognac (Francia), a pesar de la talla relativamente menor de esta última. Parece interesante consignar que en Brasil, y para sedimentos asignados al Maastrichtiano, se han ilustrado girogonites similares bajo cf. Gobichara (Pseudoharrisichara) groeberi por Musacchio (in Dias Brito et al., 2001:271, est. VIII, figs. 9-12).

Finalmente, se consigna en esta asociación la presencia de girogonites correspondientes a Lamprothamnium y Chara [estos últimos, previamente Grambastichara (in Uliana \& Musacchio, 1978:est. IV, figs. 74-76)]. Estos dos géneros mencionados en último término, no habían sido registrados en sedimentos pre-campanianos de la misma región. 

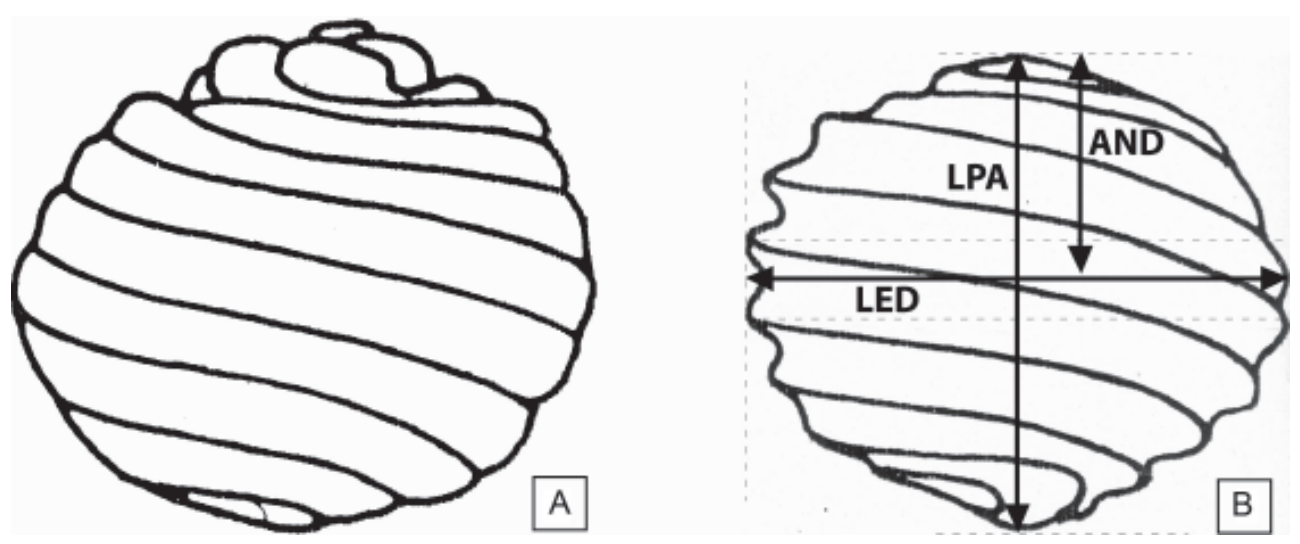

Figura 5. Platychara perlata, Fomación Yacoraite (Jujuy, Maastrichtiano): A, Girogonite fuertemente calcificado en vista lateral; B, Girogonite débilmente calcificado en vista lateral. Abreviaturas: AND, distancia anisopolar; LED, diámetro ecuatorial máximo; LPA, longitud del eje polar;. Índices de Peck (1957) y Horn af Rantzien (1959).

Figure 5. Platychara perlata from Yacoraite Formation (Jujuy Province, Maastrichtian levels): A, Gyrogonite strong calcified in lateral view; B, Gyrogonite weakly calcified in lateral view. Abbreviations: AND, anisopolar distance; LED, large equatorial diameter; LPA, large polar axis. Índices from Peck (1957) and Horn af Rantzien (1959).

Tabla 1. Dimensiones (micrones) en Platychara perlata (Maastrichtiano-Paleoceno). Mediciones en 28 girogonites procedentes de la Formación Loncoche (Maastrichtiano). Abreviaturas: nv, número de vueltas en vista lateral; LPA, longitud del eje polar; LED, diámetro ecuatorial máximo; ISI, índice de isopolaridad; ANI, índice de anisopolaridad; ce, ancho de las celulas espirales; dra, diámetro de la roseta apical; ca, ancho de las células espirales en el ápice. LPA, ISI, ANI: índices de Peck (1957) y Horn af Rantzien (1959).

Table 1. Measurements (microns) of Platychara perlata (Maastrichtian-Paleocene) obtaine from 28 gyrogonites. Abbreviations: nv, number of convolutions in lateral view; LPA, large polar axis; LED, large equatorial diameter; ISI, isopolarity index (isi = Ipa/led); ANI, anisopolarity index (ANI = AND/LPA); dra, diameter of the apical rosette; ca, width of the apical cells in the apex; ce, width of the cells in lateral view; ab, width of the basal pore (Ipa, isi, ani as in Figure 5B). Índices of Peck (1957) and Horn af Rantzien (1959).

\begin{tabular}{l|cccccccc}
\hline & nv & LPA & LED & ISI & ANI & ce & dra & ca \\
\hline Máximo & 8 & 850 & 1030 & 93 & 57 & 160 & 460 & $\mathbf{1 6 0}$ \\
Mínimo & 7 & 705 & 830 & 76 & 41 & 120 & 340 & $\mathbf{1 2 0}$ \\
Promedio & $\mathbf{7 , 5}$ & $\mathbf{8 0 4}$ & $\mathbf{9 3 0}$ & $\mathbf{8 6}$ & $\mathbf{4 9}$ & $\mathbf{1 4 0}$ & $\mathbf{3 8 0}$ & $\mathbf{1 5 0}$ \\
\hline
\end{tabular}

Tabla 2. Dimensiones (micrones) en Platychara perlata (Peck \& Reker, 1947). Mediciones en 19 girogonites procedentes de la Formación El Carrizo (Paleoceno). Abreviaturas y índices como Tabla 1.

Table 2. Dimensions (microns) in Platychara perlata (Peck \& Reker, 1947). Mediciones en 19 girogonites procedentes de la Formación El Carrizo (Paleoceno). Abbreviations and indexes in Table I.

\begin{tabular}{c|cccccc}
\hline & LPA & LED & ISI & ce & dra & ca \\
\hline Máximo & 1020 & 1285 & 90 & 225 & 575 & 200 \\
Mínimo & 680 & 715 & 68 & 150 & 515 & 150 \\
Promedio & 875 & 1075 & 79,2 & 181 & 539 & 178 \\
\hline
\end{tabular}

\section{Testando el registro del centro oeste de Argentina}

Se desprende de lo consignado en el apartado anterior, que en el centro-oeste de Argentina la diversidad en las asociaciones de carófitos previas al Campaniano es reducida cuando se las compara con las del Cretácico terminal. La pobreza en el número relativo de taxa para estas floras precampanianas no parece deberse a una falencia del registro si se compara el caso argentino con los carófitos conocidos para Brasil en las diferentes entidades del Supergrupo Baurú (Musacchio et al., 2002). En este último, la Asociación de $L$. barbosai - I. cf. riogradensis, de la Formación Adamantina (Turoniano-Santoniano), incluye hasta el presente solo dos especies de carófitos y, en cambio, 22 de ostrácodos (DiasBrito et al., 2001). El muestreo para la entidad mencionada en último término ha sido cubierto con cierto detalle para 15 localidades. En cambio, en los estratos maastrichtianos equivalentes a la Formación Marília en Minas Gerais, y contando con un muestreo solo preliminar en dos afloramientos, se han reconocido y descripto hasta el presente ocho especies asignadas a seis géneros dentro de la Asociación de Neuquenocypris minor mineira / Amblyochara sp. (Dias-Brito et al., 2001).

\section{Explicando los casos de carófitos con amplia distribución geográfica hacia el Cretácico terminal}

Algunas especies de la familia Clavatoraceae (utrículos) con amplia distribución mundial son conocidos en diversas cuencas sedimentarias de Brasil y Argentina (Musacchio, 2000) en sedimentos del Barremiano y el Aptiano. Asimismo, las faunas de ostrácodos no marinos asociados a Flabellochara harrisi durante el Aptiano en Patagonia muestran también varios casos de amplia distribución mundial (Musacchio \& Chebli, 1975; Musacchio, 2001). Vicalvi \& Carvalho (2002), han citado en Maranhão (NE de Brasil) para sedimentos del Aptiano-¿Albiano?, la presencia de girogonites comparables con Mesochara stantoni (Knowlton). 


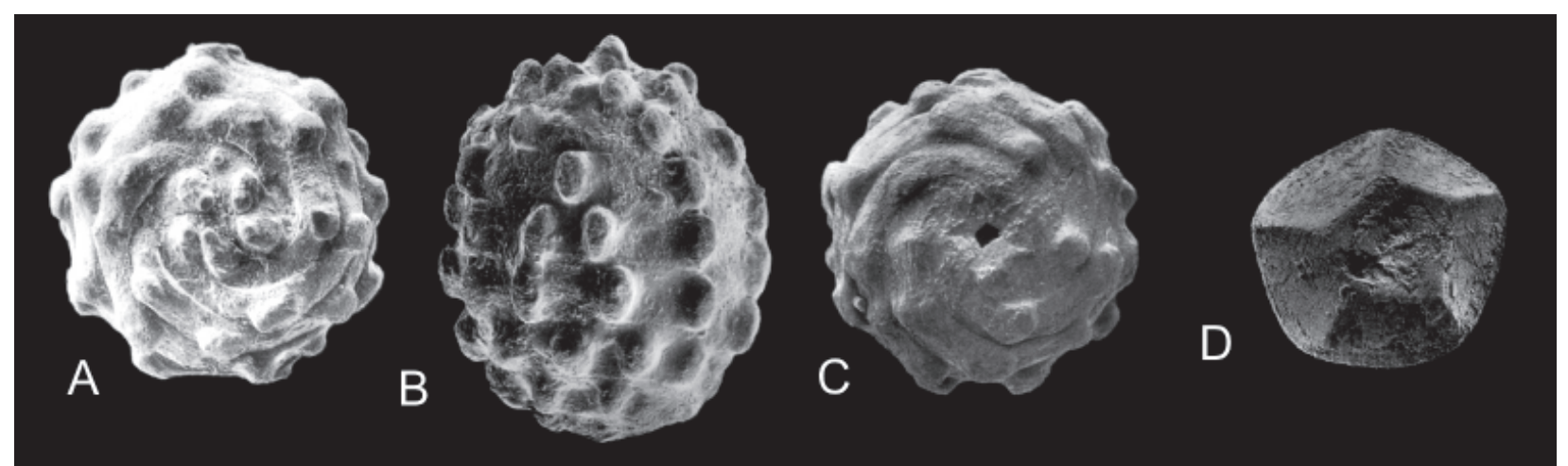

Figura 6. Peckichara cf. varians meridionalis, marcador de la asociación paleocénica homónima. Girogonites procedentes de la Formación El Carrizo: A, vista apical; B, vista lateral, LPA $=925 \mu \mathrm{m}$; C, vista basal, LED $=740 \mu \mathrm{m}$; D, placa basal, diámetro $=95 \mu \mathrm{m}$. A y B, mismo girogonite.

Figure 6. Peckichara cf. varians meridionales, a marker to the homonymous Paleocene association, from El Carrizo Formation: $\mathbf{A}$, apical view; B, lateral view, LPA $=925 \mu \mathrm{m}$; C, basal view, LED $=740 \mu \mathrm{m} ; \mathbf{D}$, basal plate, diameter $=95 \mu \mathrm{m}$. A and B, the same gyrogonite.

A partir del Barremiano Tardío-Aptiano la ruptura del aislamiento geográfico mencionado viene a modificar condiciones de endemismo que se observan en algunas asociaciones continentales neocomianas de ostrácodos y carófitos dentro de las cuencas rift. Dicha ruptura ha sido relacionada con un re-diseño paleobiogegráfico resultante de los Movimientos Miránicos iniciales (Barremiano-Eoaptiano), al menos para la faja pre-andina (Musacchio, 2000).

La ausencia de taxa con amplia distribución geográfica ("pandémicos") durante el largo período en el cual se depositaron las unidades post aptiano- ¿albianas? arriba mencionadas, va a cambiar nuevamente hacia el Cretácico terminal. En este trabajo se acepta que la Orogenia Peruana ha jugado un rol importante en esta nueva ruptura del aislamiento geográfico en América del Sur. La antigüedad de la discordancia, que data del Campaniano Temprano, ha sido documentada en los Andes Peruanos (Jaillard et al., 1993). Esta Orogenia ha permitido el intercambio geográfico de diferentes grupos fósiles permitiendo, entre otros, explicar la presencia de géneros de dinosaurios Hadrosauridae, como Kritosaurus, en Patagonia (Bonaparte, 1987).

\section{La caída en la diversidad taxonómica hacia el inicio del Terciario}

Los carófitos del Paleoceno aparecen bien representados en Mendoza y Río Negro formando una asociación con cierta identidad, la que ha permitido definir la Zona de $P$. cf. varians meridionalis Musacchio, 1989. Antes de discutir este tópico, parece necesario dar primero precisiones sobre la edad de los terrenos paleocénicos aludidos, en particular sobre los criterios disponibles para corroborar el deslinde $\mathrm{K} / \mathrm{T}$.

En diversas localidades de la cuenca, este deslinde $\mathrm{K} / \mathrm{T}$ está marcado, abajo, por diferentes microfaunas de foraminíferos planctónicos del Maastrichtiano que incluyen Heterohelicidae y Globotruncanidae (Bertels, 1980). En el Bajo del Jagüel, estas faunas están seguidas, mediando una tempestita en el K/T mismo, por una secuencia de tres asociaciones, bien preservadas, las que incluyen respectivamente los siguientes taxa de foraminíferos planctónicos marcadores con distribución global: Eoglobigerina spp., Globoconusa daubjergensis y Subottina triloculinoides. En las localidades El Zampal, El Carrizo y Pircala es posible documentar la presencia de diferentes foraminíferos que permiten establecer correspondencias con algunas de las asociaciones mencionadas (Uliana y Musacchio, 1978; Musacchio \& Moroni, 1983).

Los presentes carófitos del Paleoceno han proporcionado hasta el presente, cinco taxa: Peckichara cf varians meridionalis Massieux et al., 1981 (Figura 6), Platychara perlata (Peck \& Reker, 1947), Chara (Grambastichara) sp., Grovesichara? sp. y Lamprothamnium sp. (Musacchio \& Moroni, 1983). Las dos primeras especies son de interés regional al exhibir amplia distribución geográfica, ayudando de este modo en las correlaciones.

Corresponde, a continuación, formular y contestar la pregunta: ¿La caída en la diversidad de los carófitos conocidos en la parte meridional de América del Sur hacia el inicio del Terciario, se corresponde con la extinción masiva en el límite K/T que registran otros grupos fósiles?

En este trabajo se interpreta que esta disminución en el número de taxa para el centro oeste de Argentina parece indicar un retorno a la diversidad observada en otros intervalos del Cretácico previos al Campaniano. Los ambientes sedimentarios durante el Paleoceno no parecen aquí tan adecuados para proporcionar un registro fósil abundante como es el caso de los sedimentos tardío-cretácicos subyacentes. Estos últimos exhiben, además, diversidad en cuanto al aporte, la salinidad y el medio químico. Tal es el caso de la Formación Loncoche, la que parece haber reunido condiciones de diferentes hábitats que han sido propicios para estas algas.

\section{CONCLUSIONES}

En América del Sur meridional, el incremento en la diversidad del número de taxa que integran las floras de carófitos del Campaniano-Maastrichtiano, como así también la presencia de girogonites con amplia distribución geográfica 
dentro de las mismas, podría ser explicado en el marco de las nuevas condiciones tectónicas y sedimentarias instaladas en el Continente a partir del Campaniano Temprano.

Los carófitos del Paleoceno conocidos en sedimentos lacustres del centro-oeste de Argentina, los que suprayacen en transición a estratos marinos del Daniano con Glooboconusa daubjergensis, incluyen pocas novedades al nivel genérico cuando se las compara con las asociaciones del Maastrichtiano.

Estas asociaciones del Paleoceno parecen retornar a tasas de diversidad similares a las de las floras pre-campanianas para la misma región.

\section{AGRADECIMIENTOS}

El autor agradece a los árbitros, Mariângela Menezes (UFRGS), Valesca B. Lemos (UFRGS) y Tânia L. Dutra (UNISINOS) por las sugerencias para mejorar el original. El presente trabajo es una contribución al Proyecto plurianual Hiatos Estratigráficos (PID-UNPSJB).

\section{REFERENCIAS}

Bertels, A. 1980. Estratigrafía y foraminíferos (Protozoa) bentónicos del límite K/T en el área tipo de la Formación Jagüel. Provincia del Neuquén, República Argentina. In: CONGRESO ARGENTINO DE PALEONTOLOGÍA Y BIOESTRATIGRAFÍA, 2, 1980. Actas, Buenos Aires, p. 47-80.

Bonaparte, J. 1987. The Late Cretaceous Fauna of Los Alamitos, Patagonia, Argentina. Parts I to IX. Revista Museo Argentino de Ciencias Naturales "Bernandino Rivadavia”, Paleontología, 3(3):103-178.

Cazau, L. \& Uliana, M.A. 1973. El Cretácico Superior en la cuenca Neuquina. In: CONGRESO GEOLÓGICO ARGENTINO, 5, 1973. Actas, Buenos Aires, p. 253-280.

Dias-Brito, D.; Musacchio, E.A.; Maranhão, M.S.; de Castro, J.C.; Suárez, J.M. \& Rodrigues, R. 1998. Cretaceous non-marine calcareous microfossils from the Adamantina Formation (Baurú Group), Western São Paulo, Brasil. Boletin de la Asociación Paleontológica del Golfo San Jorge, 2:8-10.

Dias-Brito, D.; Musacchio, E.; Castro, J.C.; Maranhão, M.S.; Suárez, Rodrigues, R. 2001. Grupo Baurú: uma unidade continental do Cretáceo no Brasil - concepções baseadas em dados micropaleontológicos, isotópicos e estratigráficos. Revue Paleobiologique, 20(1):245-304.

García, A. 2003. Lychnothamnus barbatus (Meyen) Leonhardi from Australia: statistical análisis of its gyrogonite and comparison with European collections. Acta Micropaleontologica Sinica, 20(2):111-117.

Grambast, L. 1971. Remarques phylogénétiques et biochronologiques sur les Septorella du Cretacé from Provence et les charophytes associées. Paleobiologie Continentale, 2(2):1-39.

Horn af Rantzien, H. 1959. Morphological type and organ-genera of Tertiary charophyte fructifications. Stockholm Contributions in Geology, 4(2):45-197.

Jaillard, E.; Cappetta, H.; Ellenberger, P.; Feist, M.; GrambastFessard, N.; Lefranc, J.P. \& Sige, B. 1993. Sedimentology, palaeontology, biostratigraphy and correlation of the Late CretaceousVilquechico Group of southern Perú. Cretaceous
Research, 14:623-661.

Karczeewska, J., \& Ziembinska-Tworzydlo, M. 1972. Lower Tertiary Charophyta from the Nemegt Basin, Gobi Desert. Paleontologica Polonica, 27:51-81.

Kumar, A. \& Grambast-Fessard, N. 1984. Maastrichtian charophyte gyrogonites from Jamaica. Micropaleontology, 30(3):263-267.

Li, W-T. 1998. Les Charophytes de la Formation Paomagag de Danagyang (Chine). La limite Cretace/Tertiaire ages des intertraps du Deccan a Nagpur, Inde et de l'union de l'Índe a l'Ásie. Beijing, Editions de l'Industrie Petroliere, 227 p.

Massieux, M.; Tambareau, I. \& Villate, J. 1981. Charophytes thanétienes et ilerdienes de la coverture accidental du Massif de Monthounet (Aude). Géologie Méditerranéenne 8(1):1-17.

Musacchio, E.A. 1973. Charophytas y ostrácodos no marinos del Grupo Neuquén (Cretácico Superior) en algunos afloramientos de las Provincias de Río Negro y Neuquén en la República Argentina. Revista del Museo de La Plata, n.s., Paleontología, 8:1-33.

Musacchio, E.A. 1989. Biostratigraphy of the non-marine Cretaceous of Argentina based on calcareous microfossils. In: J. Weidmann (ed.) Cretaceous of the Western Tethys, E. Schweizerbart'sche Verlagsbuchandlung, p. 811-850.

Musacchio, E.A., 2000. Biostratigraphy and biogeography of Cretaceous Charophyta from South America. Cretaceous Research, 21:211-220

Musacchio, E.A., 2001. Relaciones paleobiogeográfícas de los ostrácodos no marinos del Jurásico y el Cretácico de Patagonia. Acta Geológica Leopoldensia, 24(52/53):293-310.

Musacchio, E.A. \& Chebli, G. 1975. Ostrácodos no marinos y carófitos del Cretácico Inferior en las Provincias del Chubut y Neuquén en Argentina. Revista de la Asociación Paleontológica Argentina, 12(1):70-96.

Musacchio, E.A. \& Moroni, A.M. 1983. Charophyta y Ostracoda no marinos eoterciarios de la Formación El Carrizo en la Provincia de Río Negro, Argentina. Ameghiniana, 20(1):21-33.

Musacchio, E.A. \& Simeoni, M. 1991. Taxonomy of some Cretaceous non-marine ostracods of palaeobiogeographical interest. Neues Jahrbuch für Geologie und Palaöntologie, Abhandlungen, 180(3):349-389.

Musacchio, E.A.; Dias-Brito, D.; Maranhão, M.S. \& Suárez, J.M. 2002. Comparando carófitos (algas verdes) neocretácicos del Grupo Baurú (Brasil) y de Argentina. In: SIMPÓSIO SOBRE O CRETÁCEO DO BRASIL, 6 / SIMPOSIO SOBRE EL CRETÁCICO DE AMÉRICA DEL SUR, 2, 2002. Resumos Expandidos, Rio Claro, p. 229-234.

Peck, R.E. 1957. North American Mesozoic Charophyta. U.S.A. Geological Survey, Professional Paper, 294(A):1-44.

Peck, R.E \& Reker. C.C. 1947. Cretaceous and Lower Cenozoic charophytes from Perú, South America. American Museum Novitates, 1369:1-6.

Petri, S. 1955. Charophyta cretácicas de São Paulo (Formacão Baurú). Boletim da Sociedade Brasileira de Geologia, 4(1):67-72.

Soulie-Märsche, I. 1989. Etude comparée de gyrogonites de Caharophytes actuelles et fósiles et philogenie des genres actuels. Université des Sciences et Techniques du Languedoc, Montpellier, Thêse d'Etat, 237 p.

Soulie-Märsche, I. \& Martin-Closas, C. 2003. Lychnotamnus barbatus (charophytes) from the Upper Miocene of $\mathrm{La}$ Cerdanya (Catalonia Spain): taxonomic and palaeoecological implications. Acta Micropaleontologica Sinica, 20(2):156-165.

Uliana, M.A. \& Musacchio, E.A. 1978. Microfósiles calcáreos no 
marinos del Cretácico Superior en El Zampal, Provincia de Mendoza, Argentina. Ameghiniana, 15:11-135.

Vallati, P., 2001. Middle Cretaceous microflora from the Huincul Formation ("Dinosaurian Beds") in the Neuquén Basin, Patagonia, Argentina. Palynology, 25:179-197.

Vicalvi, M.A., Carvalho, I. 2002. Carófitas cretáceas da Bacia da Paranaíba (Formação Itapecurú) Estado do Maranhão, Brasil. In: SIMPÓSIO SOBRE O CRETÁCEO DO BRASIL, 6 / SIMPOSIO SOBRE EL CRETÁCICO DE AMÉRICA DEL SUR, 2, 2002. Resumos Expandidos, Rio Claro, p. 229-234.
Wang, Z. 1978. Cretaceous Charophytes from the Yangze-Han river basin with a note on the classification of Porocharaceae and Characeae. Memoirs of the Nanjing Institute of Geology and Paleontology, Academia Sinica, 9:61-88 (en chino).

Weaver, C., 1931. Paleontology of the Jurassic and Cretaceous of WestCentral Argentina. University of Washington Memoir 1, $469 \mathrm{p}$.

Received in January, 2006; accepted in March 2006. 